\title{
Alpers- and MNGIE-like disease with disturbed CSF folate transport and an unusual mode of genetic transmission of POLG mutations: a case report.
}

\author{
Rudolf Korinthenberg $^{1(\mathbb{D})}$; Janbernd Kirschner ${ }^{2}{ }^{(\mathbb{D})}$; Matthias Eckenweiler ${ }^{1(\mathbb{D})}$, Robert Steinfeld $^{3}$,

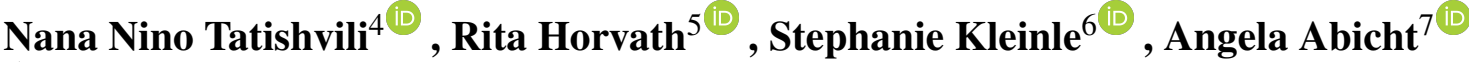 \\ ${ }^{1}$ Department of Neuropediatrics and Muscle Disorders, University Medical Center, Faculty of Medicine, University of Freiburg, \\ Germany \\ ${ }^{2}$ Department of Neuropediatrics, University Hospital Bonn, Bonn/Germany \\ ${ }^{3}$ Department of Neuropediatrics, University Children's Hospital Zurich, Zurich/Switzerland \\ ${ }^{4}$ M. Iashvili Childrens Central Hospital, Tbilisi/Georgia \\ ${ }^{5}$ Department of Clinical Neurosciences, University of Cambridge, Cambridge, UK \\ ${ }^{6}$ Medical Genetics Centre, Munich/Germany \\ ${ }^{7}$ Medical Genetics Centre, Munich/Germany and Friedrich-Baur-Institute, Dept. of Neurology, LMU Klinikum, Munich/Germany \\ Corresponding author: Rudolf Korinthenberg; rudolf.korinthenberg@ uniklinik-freiburg.de
}

do) https://doi.org/10.17724/jicna.2020.216

Received: 2 November 2020

Accepted: 9 September 2021

\begin{abstract}
We report on a family where three out of five siblings were affected by a variable remitting-relapsing disease presenting with epileptic seizures, coma and abdominal crises with lethal outcome. In the youngest son and one of his deceased brothers, we identified two disease-causing compound heterozygous $P O L G$ variants. One of these variants was inherited from the mother, but the other was absent in the father's blood, saliva, buccal swab, and hair bulbs, although his paternity was genetically proven. We thus assume germline mosaicism for this mutation in the father. Very low 5-methyltetrahydrofolate (5-MTHF) and absence of folate receptoralpha were repeatedly found in the CSF of the youngest brother, indicating a secondary cerebral folate transport deficiency. Folinic acid supplementation for 18 months resulted in some temporary improvement of the neurological symptoms, but failed to prevent systemic deterioration and death.
\end{abstract}

Keywords: Polymerase gamma gene, Alpers-Huttenlocher Syndrome, MNGIE, gastric dysmotility, CSF 5-MTHF, folinic acid.

(C) Korinthenberg R. et al.; licensee JICNA

\section{Introduction}

The POLG gene codes for the catalytic alpha-subunit of polymerase-gamma, which is involved in mitochondrial DNA (mtDNA) replication. Pathogenic POLG-variants can lead to mtDNA-depletion or mtDNA-deletions associated with an extremely broad spectrum of different clinical phenotypes. Biallelic pathogenic variants in POLG usually result in mtDNAdepletion and autosomal-recessive early-onset diseases such as infantile and early-lethal multisystem myocerebrohepatopathy spectrum (MCHS) disorder or Alpers-Huttenlocher syndrome (AHS). These conditions present in the first years of life with epileptic encephalopathy, myoclonic seizures, ataxia, hepatopathy and profound developmental regression [1, 2, 3]. A milder phenotype has been associated with less severe mtDNA changes and later onset of symptoms, such as ataxia, peripheral neuropathy and seizures in adolescents and young adults, and late-onset progressive external ophthalmoplegia (PEO) and ataxia in adults more thn 40 years [4].
Here we report three siblings who were affected by a progressive epileptic encephalopathy leading to coma, abdominal crises and death. We identified two rare compound-heterozygous $P O L G$ variants in the affected children, which were predicted to be damaging. One of the variants was probably transmitted by germline mosaicism in the father. Very low CSF-5methyltetrahydrofolate (5-MTHF) and absence of CSF-folate receptor-alpha indicated a secondary cerebral folate transport deficiency as a further debilitating feature in the one sibling investigated at our centre. Folinic acid supplementation resulted in some transient improvement of the neurological condition; however, it did not prevent overall disease progression and death. 


\section{Case report}

\section{Family history}

The index patient was the youngest of five children of nonconsanguineous parents of ethnic Georgian origin. The mother was reported to be healthy; the father suffered from severe migraines. The oldest son of the family, currently 29 years old, was in good health. The 23-year-old daughter reported chronic gastrointestinal complaints but did not have neurological problems and had a healthy three-year-old son. All three younger siblings developed progressive neurological symptoms in early childhood: the first affected son developed normally until the age of five years when he suffered focal myoclonic jerks in the right hand, followed by speech arrest and right arm weakness. These symptoms resolved after two weeks, and he regained the ability to ride a bicycle. However, three months later a second episode occurred with severe headache and abdominal cramps. As appendicitis was ruled out and liver enzymes were normal he was dismissed from the hospital. On the following day, he appeared pale and stopped speaking. A few hours later, acute deterioration with somnolence and coma occurred; he died 24 hours later in a Russian hospital without an exact diagnosis. Imaging or lactate findings from this patient were not available. The second affected son also developed normally up to the age of five years. Then, unexpectedly, he lost consciousness and presented rightsided hemiparesis; a cranial MRI showed normal findings. A second episode occurred at seven years of age: in the course of an unspecific pneumonia he transiently lost consciousness and vision. Suspecting epileptic seizures, he was treated with carbamazepine, but his EEG was reported to be normal. An MRI showed non-specific changes of the bilateral occipital white matter. His third episode occurred at the age of 10 years. After an unspecific illness, focal convulsive status epilepticus (epilepsia partialis continua) occurred with deviation of head and eyes to the right and twitching of the right side of the face, accompanied by left-sided focal spike and slow-wave activity. Liver enzymes and all other blood tests were normal. The MRI was unchanged. After prolonged treatment with thiopentone-anaesthesia at a Georgian hospital, he was alert and responsive for three days. However, acute severe deterioration occurred, and the boy died within 10 hours due to recurrent status epilepticus, acidosis, anuria and respiratory failure.

\section{History and findings of the index case}

History. The third affected son of the family was born prematurely after a normal pregnancy at the $35^{\text {th }}$ week of gestation but without further complications. His development in infancy was normal: he sat up at six months, walked independently at 14 months, and spoke his first words at 10 months. A first episode of vomiting and abdominal cramps occurred at the age of eight years and four months. These symptoms ceased after two weeks, but his muscular strength remained somewhat reduced. A second episode occurred one year later. Following an onset with vomiting and abdominal pain, micturition ceased, his consciousness deteriorated, he became comatose, and he required mechanical ventilation for three weeks. Laboratory values reported from the Georgian hospital showed normal CSF-lactate and serum transaminases, borderline raised creatine kinase $(\mathrm{CK})$ and hypernatraemia, low free carnitine $(1.1 \mathrm{mg} / \mathrm{dl}$, a lower limit of normal $3.5 \mathrm{mg} / \mathrm{l}$ ), and significantly raised acetoacetate. $\mathrm{He}$ slowly recovered after this episode with residual muscle weakness, but was unable to walk for longer distances. He was unable to attend school but was educated by a private teacher.

Clinical presentation. Living in Georgia, the boy was presented at Freiburg University Medical Center (FUMC), Germany, for the first time at the age of 10 years and four months. At the initial clinical evaluation, he appeared alert and interacted adequately. However, no formal cognitive testing was performed. He was short for his age and underweight (height $118 \mathrm{~cm}(0.1$ percentile), weight $16.2 \mathrm{~kg}$ (0.3 percentile), body mass index (BMI) 11.7 (-4.2 SDS). He looked pale, but his cardiovascular system, lungs, abdominal organs and skeleton appeared normal. With the exception of a very slight ptosis, cranial nerve function was normal, and there was no ophthalmoplegia or nystagmus. His muscle tone and strength were moderately reduced. Deep tendon reflexes (DTR) were normal at the upper limbs, whereas they were not elicitable at the lower limbs. The Babinski sign was negative. His gait was moderately ataxic, with a slightly positive Romberg sign. Standing and hopping on one leg were possible only with support, and his tandem gait was grossly abnormal. His Gowers' sign was positive, and time to rise from supine was 25 seconds. He showed bilateral dysdiadochokinesis, but the finger-nose test was without abnormalities.

Specialist investigations. Ophthalmological investigations revealed a pale fundus with some abnormal pigmentation, but eye movements, electroretinography (ERG) and perimetry were normal. Ears, nose and throat (ENT) investigations revealed normal auditory function.

Radiological and ultrasonographic investigations. A cranial MRI at the age of 10 years and four months showed diffuse T2-hyperintense lesions in the deep white matter of both hemispheres with sparing of U-fibers. There was also a subtle increased T2-signal in the caudate heads and putamen (Figure 1). Diffusion-weighted imaging was normal and there was no abnormal contrast-enhancement. A magnetic resonance spectroscopy (MRS, PRESS $30 \mathrm{~ms}$ ) of the basal ganglia revealed normal findings without an elevated lactate peak or other abnormal peaks. $\mathrm{NAA} / \mathrm{Cr}$ and $\mathrm{Cho} / \mathrm{Cr}$ ratios were within normal limits. An MRS of occipital white matter was not performed. An X-ray of the left hand showed delayed skeletal maturation (seven years and +/- 11 months at the age of 10 years and four months) and osteopenia. Ultrasonographic investigations of heart, thyroid and abdominal organs showed no abnormalities.

Further technical investigations. Spirometry was conducted without abnormalities. Electroencephalogram (EEG) background activity during waking was normal for his age, and there were no epileptic potentials or focal slowing. Nerve conduction studies gave evidence of a diffuse sensorimotor neuropathy with moderate slowing of motor and sensory nerve conduction velocities (mNCV, sNCV) and low amplitudes (A): median nerve mNCV 35 (normal [n] > 50) m/s, A $2.0(n>4) \mathrm{mV}$; tibial nerve 
mNCV $31(n>40) \mathrm{m} / \mathrm{s}, \mathrm{A} 4.2(\mathrm{n}>2) \mathrm{mV}$; median nerve sNCV $39(\mathrm{n}>50) \mathrm{m} / \mathrm{s}, \mathrm{A} 2.5(\mathrm{n}>15) \mu \mathrm{V}$; sural nerve NCV $34(\mathrm{n}>50)$ $\mathrm{m} / \mathrm{s}, \mathrm{A} 4.8(\mathrm{n}>20) \mu \mathrm{V}$.

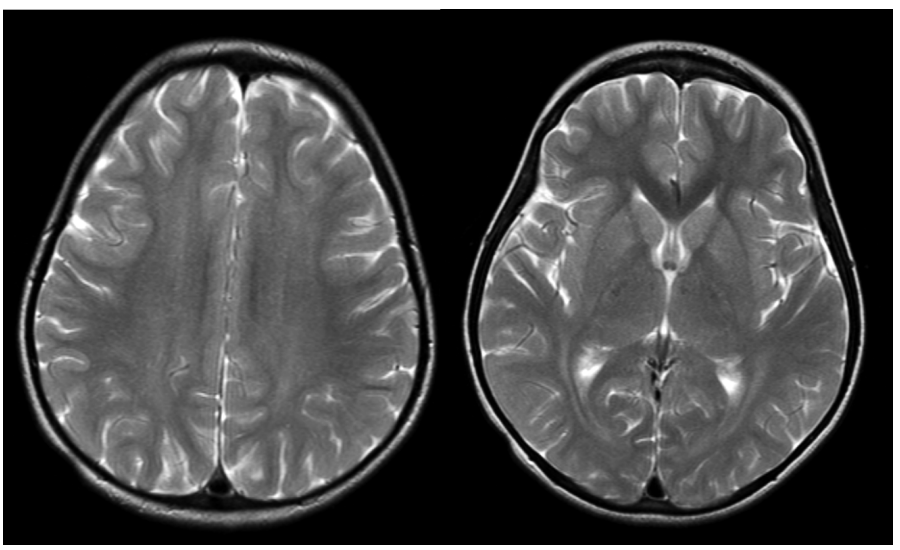

Figure 1. T2-weighted MRI in our index-case at the age of 10.6 years. The MRI shows an increase in T2 signal intensity in the white matter, diffusely in the centrum semiovale and periatrial white matter, but sparing of the U-fibres and the optic radiations. There is also a subtle increase in the T2 signal in the caudate heads and putamen.

Clinical and metabolic laboratory investigations. The following findings were within normal limits:

- Whole-blood (EDTA, heparin, citrate): red and white blood cell count, plasma-ammonia, plasma amino acids, Prothrombin Time (Quick) and partial thromboplastin time (PTT).

- Serum: electrolytes, creatinine, uric acid, liver transaminases, gamma-GT, CK, thyroid hormones and thyroid antibodies, vitamin A, -B1, -B2, -B6, -E and -B12, folic acid, isoelectric focusing of sialotransferrins and pancreasamylase.

- Urine: amino acids and organic acids, D-aminolaevulinic acid, porphobilinogen and total porphyrins.

- Faeces: elastase and Helicobacter-antigen.

- A 24 hour-fasting test showed normal levels of acylcarnitines, and a normal rise of ketone bodies in plasma, normal blood lactate and ammonia, and normal ketone bodies, organic acid and amino-acid concentrations in urine.

The following abnormal results were found:

- Serum 25-OH-Vitamin D2 was low (10.3 ng/ml, reference range $20-70 \mathrm{ng} / \mathrm{ml}$ ), and bone alkaline phospatase increased $(62.4 \mu \mathrm{g} / \mathrm{l}$ - normal range 6-30), parathormone was not investigated.

- Basal serum lactate was normal (1.1-1.6 mmol/l); two hours after an oral glucose load, an abnormal increase to $3.11 \mathrm{mmol} / \mathrm{l}$ (lower limit of normal $+20 \%$ over basal value) was noted. Also, serum lactate was found to increase (3.0 $\mathrm{mmol} / \mathrm{l}$ ) repeatedly during night-time vomiting.
Investigations of lumbar CSF. Cell count, glucose and blood/CSF glucose ratio, and lactate $(2.4 \mathrm{mmol} / \mathrm{l})$ were normal (normal $<2.5 \mathrm{mmol} / \mathrm{l}$ ). CSF-protein was distinctly increased with $350 \mathrm{mg} / \mathrm{dl}$ (normal < $50 \mathrm{mg} / \mathrm{dl}$ ). The blood-brain barrier was impaired, but there was no intrathecal IgG-synthesis or oligoclonal bands. CSF-HVA, -5-HIAA and -neopterin were insignificantly increased, CSF-tetrahydrobiopterin was slightly low, CSF-dihydrobiopterin normal. However, 5-MTHF was distinctly decreased (5 nmol/1 - normal range 64-182), and at a repeated lumbar puncture four weeks later was below detectable levels $(<2 \mathrm{nmol} / \mathrm{l})$. Western blot showed reduced folate receptoralpha $(\operatorname{Fr} \alpha)$ protein levels (Figure 2). Low CSF-5-MTHF, together with normal serum and red blood cell folate, and also reduced Fr $\alpha$ are typical findings in primary or secondary defects of CSF-folate transport [5] (see discussion).

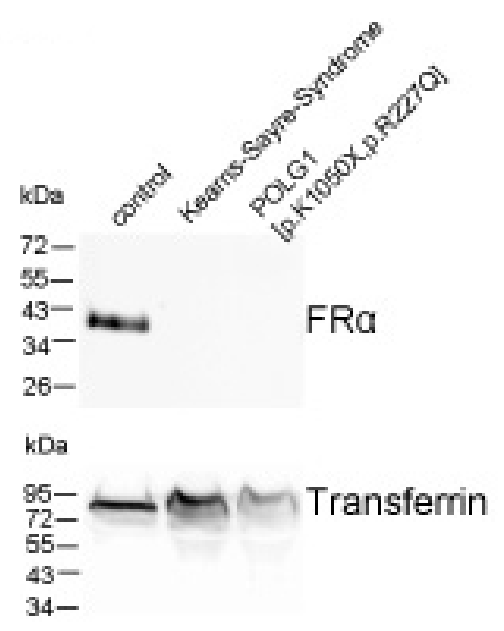

Figure 2. Western-Blot of Folate Receptor-alpha in the CSF of our patient as compared to a healthy control and a case with Kearns-Sayre syndrome.

\section{Treatment and further clinical course of the index case}

The patient remained an out-patient at FUMC for four months. Suspecting mitochondrial disease, we initially started treatment with $100 \mathrm{mg}$ thiamine, $80 \mathrm{mg}$ carnitine, and $100 \mathrm{mg}$ coenzyme Q10 in single doses per day. (Later, an urgent request from the parents stopped us from discontinuing these drugs). Furthermore, we applied 1,000 IU vitamin D/day. About eight weeks later, after confirmation of an impaired CSF-folate transport, we 
added high-dose oral $(2.8 \mathrm{mg} / \mathrm{kg} /$ day three times a day [tid] $)$ and intravenous (iv, $1 \times 6.2 \mathrm{mg} / \mathrm{kg} /$ week) calcium folinate (leucovorin) as proposed for treatment of both the hereditary and acquired transport defects $[5,6]$. At a repeated lumbar puncture, after four weeks of treatment with oral (p.o.) high-dose plus intravenous leucovorin, the CSF-5-MTHF concentration had normalised (128 nmol/l). Over several weeks, the parents observed improvement in endurance and strength. The boy was now able to climb stairs without the help of his hands and used a wheelchair much less frequently. The frequency of abdominal cramps decreased, and his mood improved. At a final clinical investigation before returning to Georgia (at 10 years and eight months of age), his height had increased by $2 \mathrm{~cm}$ and his weight by $0.8 \mathrm{~kg}$. His gait was broad-based but no longer ataxic. Walking on his heels and toes was possible with some difficulty. He was able to stand on one leg (left for 4-5 seconds, right 2-3 seconds). His tandem gait was unsteady; hopping on one leg was not possible without support.

Back in Georgia a few months later, despite unchanged medication, the abdominal pain and vomiting recurred with increasing frequency and the boy's general condition steadily deteriorated. When necessary, however, he could still walk a few hundred meters.

Follow-up investigations and treatment at FUMC took place one year later (aged 11 years and seven months). Epigastric cramps of 10 minutes to one hour duration occurred daily, also during the night. He vomited four to five times per week and felt better afterwards. An oesophago-gastro-duodenoscopy revealed an atonic stomach with biliary reflux from the duodenum, and secondary unspecific gastritis; no ulcer or passage obstruction was detected. Symptomatic treatment and frequent small meals were recommended. The findings of a repeat cranial MRI were unchanged from the preceding year. Two follow-up investigations of lumbar CSF were performed. The first, after continuous treatment with high-dose (oral plus intravenous, as above, body weight $15.5 \mathrm{~kg}$ ) leucovorin for one year, showed a CSF 5MTHF concentration persistently within the normal range. At the second investigation, three months after discontinuing the intravenous injections and continuing leucovorin with $45 \mathrm{mg}$ p.o./day tid, CSF-5-MTHF still remained in the normal range (70.8 nmol/l, normal $64-182 \mathrm{nmol} / \mathrm{l})$. Therefore, we continued treatment with oral leucovorin $45 \mathrm{mg} /$ day tid; the parents also insisted on continuing L-carnitine $80 \mathrm{mg}$ and high-dose vitamins as described above.

At the last investigation at FUMC, at the age of 11 years and 11 months, the boy's health seemed somewhat improved. The abdominal cramps had decreased, but he still vomited once per day. However, he appeared severely cachectic (height $124 \mathrm{~cm}$, weight $15.5 \mathrm{~kg}$ ) with distinct muscular atrophy. His strength seemed adequate for this condition; there were no pareses. There was mild facial weakness and ptosis, but no other cranial nerve defects, no nystagmus. His gait was unsteady and the Romberg sign was positive. Standing and hopping on one leg were no longer possible, even with support. Muscle tone appeared slightly reduced, but DTRs were elicitable normally. The Babinski sign was negative.
Back home in Georgia, he deteriorated rapidly despite continued treatment. From the age of 12 years and three months he was repeatedly admitted to the local hospital due to vomiting and intestinal dysmotility. Enteral nutrition was no longer possible, and intravenous parenteral nutrition was initiated. This was complicated by repeated infections, respiratory insufficiency with multiple pulmonary abscesses, septic shock, anuria and dialysis. As yet, no hepatopathy was noted. Eventually, the boy died at the age of 12 years and six months; no epileptic fits or unresponsive episodes had occurred from the age of 10 years until death.

\section{Muscle biopsy and genetic investigations}

Muscle biopsy. In the index case, a muscle biopsy from the lateral vastus muscle showed very slight myopathic changes on light-microscopy (hematoxylin-eosin [HE] and trichrome stains). On histochemical staining an increase of COXnegative/SDH-positive fibres, as well as a prominent smalldroplet intracellular lipid accumulation (Oil Red O), were found. No neurogenic changes, no inflammatory infiltrates, and no increased glycogen accumulation were seen.

Investigation of the activity of the respiratory chain complexes was performed in skeletal muscle homogenate as previously described [7] and detected normal activities of complexes I-III, but a borderline low activity of Complex IV (103.1 U/gNCP; normal range $112-351 ; 1.07$ related to citrate synthase; normal range 1.1-5.0).

Material for genetic investigations. We were able to study muscle DNA of the index patient, as well as DNA extracted from peripheral blood of the index case, his healthy brother, and the parents. A dried blood spot of one of the deceased siblings contributed DNA for that case. In addition, we studied paternal DNA extracted from blood, saliva, buccal swab, and hair bulbs. The sister refused genetic testing in herself and her son.

Muscle DNA. In a semiquantitative analysis by a real-time polymerase chain reaction (PCR) test [8], muscle DNA of the index patient revealed reduced mtDNA copy numbers, suggestive of mtDNA depletion (20-30\% of mtDNA, relative to nuclear DNA compared to controls). No mtDNA deletions were found on standard diagnostic testing with long-range PCR and Southern blot.

Polymerase-gamma-gene (POLG) analysis. Sanger sequencing of all coding regions of $P O L G$ with exon-intron boundaries in blood DNA of the index patient detected two heterozygous variants, while MLPA analysis was normal. One of these NM_002693.2: c.680G > A p.(Arg227Gln) is a missense variant that has recently been described in three infants with mitochondrial depletion syndrome (MDS) and Alpers-Huttenlocher syndrome (AHS) $[9,10,11]$. This variant was also present in blood DNA of the deceased brother but not in the parents and the healthy brother. A buccal swab, saliva and hair bulbs of the father were also negative for this mutation. Paternal blood DNA was additionally tested by deep targeted next-generation sequencing (coverage $>2500$ ) for this POLG variant and was negative. However, paternity was confirmed by genetic markers investigated in both parents and the index case. The second variant c.3148A > T 
p.Lys $1050 *$ that has not yet been described in the literature gives rise to a stop-codon; it was detected in the index patient and the deceased brother, and was also found in the mother's blood, but not in that of the father and the healthy eldest brother. These data suggest the presence of paternal somatic or, most probably, germline mosaicism for the c.680G > A p.(Arg227Gln) (see discussion).

\section{Discussion}

\section{Significance and transmission of the POLG variants}

The POLG gene at chromosomal location $15 \mathrm{q} 26.1$ codes for the catalytic alpha subunit of the polymerase-gamma. Together with additional proteins, polymerase-gamma is involved in replication of the single-stranded mitochondrial DNA (mtDNA). Pathogenic variants, if biallelic, may result in severe early onset autosomal-recessive disorders with mtDNA depletion, while heterozygous variants cause later-onset autosomal-dominant disorders with multiple mtDNA deletions $[1,2,3,4]$.

In our index case, we found two heterozygous variants in the POLG gene, the missense variant c.680G > A p.(Arg227Gln) and the stop variant c.3148A $>$ T p.Lys 1050*. The sequence change c.3148A $>\mathrm{T}$ creates a premature translational stop signal (p.Lys1050*) in the POLG gene. Since this premature termination codon (PTC) is not located in the last or penultimate exon of $P O L G$, nonsense-mediated decay (NMD) of mRNA can be assumed which is expected to result in an absent protein product. The variant c.3148A > T p.Lys1050* is not present in population databases (ExAC no frequency) or clinical databases (ClinVar). It was classified as pathogenic (class 5: evidence codes PVS1, PM2, PM3, PP3) according to the ACMG guidelines [12]. The missense variant p.Arg227Gln results from a $\mathrm{G}$ to A substitution at nucleotide position 680. This nucleotide change is absent in population databases (ExAC no frequency). However, the resulting amino acid change from arginine to glutamine at codon 227, which occurs at a position that is conserved across species, has been reported in clinical databases (ClinVar) as pathogenic and as compound heterozygous in several individuals with $P O L G-$ related disorders. Bioinformatic algorithms predict a potential impact of the missense change on protein structure and function (SIFT: 'Deleterious'; PolyPhen-2: 'Probably Damaging'). Based on this evidence, this variant was classified as pathogenic (class 5: evidence codes: PS1, PM2, PM3, PP1, PP2, PP4) according to the ACMG guidelines [12]. The pathogenicity of both variants is further supported by the finding of the mtDNA depletion in the muscle biopsy of our index case.

Segregation analysis in the family (Figure 3) supported pathogenicity by revealing that the index patient and one affected sibling each carried both heterozygous variants. There was no DNA available from the third affected brother. The healthy brother did not carry any of the two variants. Consistent with autosomal recessive inheritance, the healthy mother was found to be a heterozygous carrier for one of the variants (c.3148A $>\mathrm{T}$ p.Lys1050*). However, the variant c.680G > A p.(Arg227Gln) was absent from the father's blood sample. Paternity of the father could be confirmed by genetic markers investigated in both parents and the index case.

Searching for somatic mosaicism of variant c.680G > A p. (Arg227Gln) in the father, additional tissues (buccal swab, saliva and hair bulbs) were investigated by targeted Sanger sequencing but were found to be negative. As Sanger sequencing is known to have a detection limit of $10 \%$ to $20 \%$ [13], low-grade somatic mosaicisms might only be detectable by more sensitive methods. It could be more frequent than previously assumed as deep sequencing of parental samples from children with de novo variants in genes causative of epileptic encephalopathy revealed low levels of somatic mosaicism ranging from $1-10 \%$ in blood in 5-6\% of cases [14, 15]. However, as deep sequencing was negative in our patient's father, we rather suggest a germline mutation as the underlying cause. Germline mutations giving rise to affected siblings in apparent non-carrier parents are well known in autosomal-dominant and X-linked diseases. However, parental somatic or germline mosaicism in autosomal-recessive conditions have rarely been described $[16,17]$.

Discussion of the multiple phenotypes in this family It is known that pathogenic variants in the $P O L G$ gene can lead to multiple phenotypes. In young infants with mtDNA depletion, myocerebrohepatopathy spectrum (MCHS) disorder is the earliest occurring and most severe phenotype. It is followed by Alpers-Huttenlocher syndrome (AHS), which leads to death by the end of the first decade $[1,2,3,4,18]$. In contrast, diseases associated with mtDNA deletions, such as myoclonic epilepsy myopathy sensory ataxia (MEMSA), ataxia neuropathy spectrum (ANS, SANDO) disorders, autosomal recessive (arCPEO), and autosomal dominant progressive external ophthalmoplegia (adCPEO), occur in older age groups [4]. It is important to note that there is a strong overlap between these phenotypes, most of which were described before the discovery of the common genetic etiology. The following additional symptoms and signs have been reported to occur in AHS and pathogenic $P O L G$ variants: failure to thrive, Leigh syndrome with dystonia and pathology of basal ganglia and brainstem, migraine attacks, stroke-like episodes with and without MRI changes (similar to MELAS), pancreatitis and gastrointestinal dysmotility and pseudo-obstruction similar to mitochondrial neurogastrointestinal encephalomyopathy (MNGIE) [1, 2, 3, 4]. Tang et al. found four patients with MNGIE-like disease in a cohort of 92 patients with $P O L G$ variants when reevaluating clinical data; their age at diagnosis was 15, 25, 46 and 50 years. POLG-MNGIE could be differentiated from typical TYMP-MNGIE by the lack of CNS leucoencephalopathy [19].

Several of these syndromes and symptoms were found in the family presented here, but with considerable variability between the affected brothers. A common feature was the relapsing, repetitive character of the neurological and systemic symptoms. The symptoms in the older brothers with recurring epilepsia partialis continua and encephalopathy could be well classified as Alpers disease; similar to our index case, they showed no liver involvement $[1,2,3,4,18]$. Their periodic episodes with coma or hemipareses appeared to be stroke-like episodes, de- 


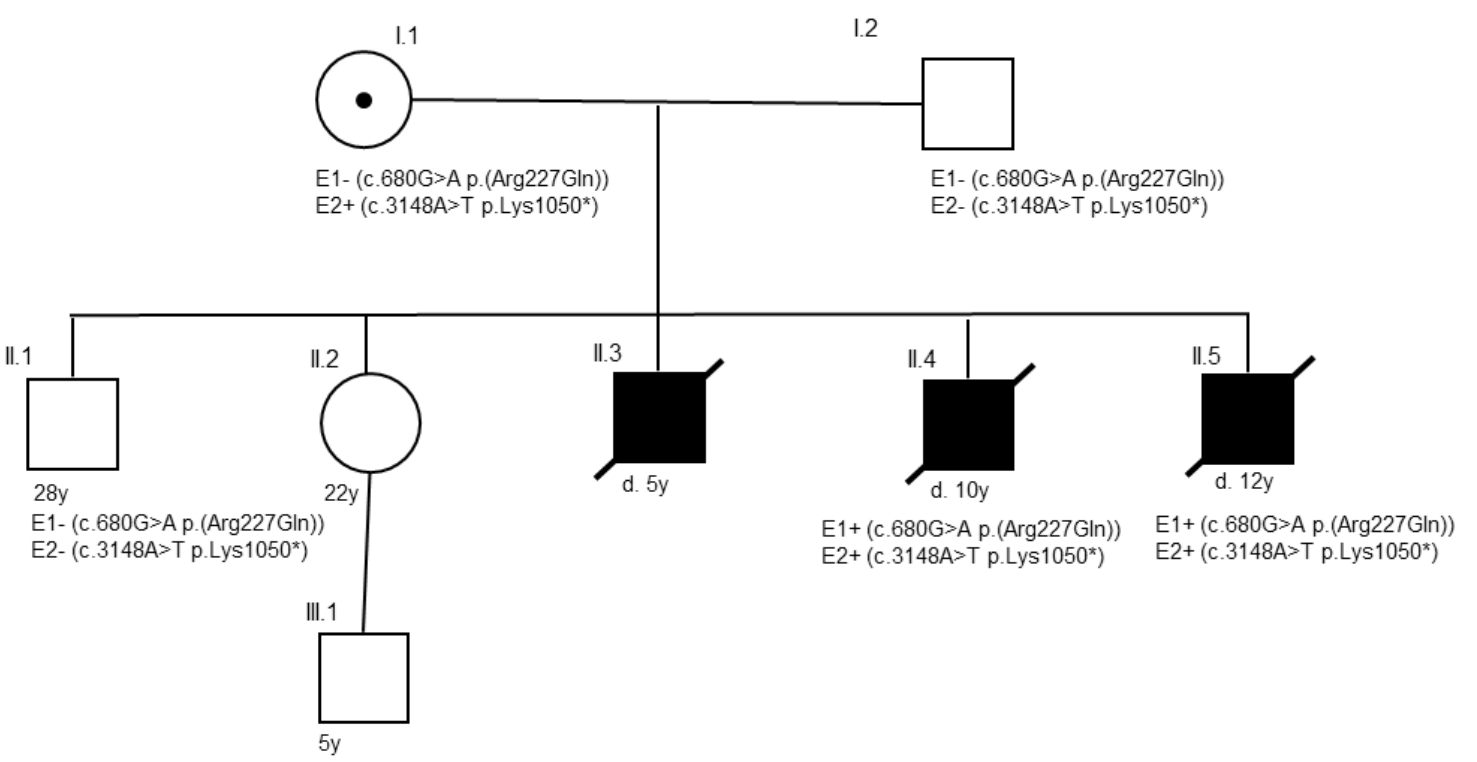

Figure 3. Pedigree of the reported family showing the segregation of the POLG mutations.

spite very unspecific MRI findings. In our index case, however, failure to thrive and gastro-intestinal complaints dominated the clinical picture, along with mild retardation, subtle ataxia, and polyneuropathy. Early in the course of disease, he suffered a severe stroke-like episode with normal MRI, but he never experienced seizures or showed epileptic potentials on EEG. Increasing abdominal cramps, vomiting, and gastro-intestinal dysmotility and its complications prevailed in the later course. Hence, the most appropriate clinical diagnosis for our index case would be $P O L G$-related MNGIE syndrome.

Significance and mechanism of CSF-folate depletion and discussion of treatment

Activated folate is an important cofactor in nucleic acid biosynthesis, one carbon metabolism and amino acid metabolism, and thus has an essential role in cell-proliferation and blood-cell regeneration, as well as in CNS metabolism and CNS-function. Decreased CSF 5-MTHF may be a consequence of nutritional folate deficiency or inherited intestinal folate malabsorption (for example due to pathogenic variants in the SLC46A1 gene); in both conditions low blood folate levels in serum, red blood cells and CSF are found. But low CSF 5-MTHF may also be due to impaired transport at the bloodCSF-brain barrier despite normal serum and red blood cell folate. A progressive neurological syndrome in young children has been published due to low CSF 5-MTHF as a consequence of antibody-mediated folate receptor dysfunction [20]. Steinfeld et al. first described a genetic syndrome due to pathogenic variants in the folate receptor-1 gene (FOLR1), leading to folate receptor-alpha $(\mathrm{FR} \alpha)$ deficiency. Their patients suffered progressive movement disturbance, psychomotor regression, ataxia and myoclonic epilepsy. The MRI showed profound hypomyelination, and MRS indicated a combined depletion of white-matter choline and inositol [6]. The same group demonstrated that folate transport via the blood-CSF barrier is mediated by folate receptor-alpha dependent transcytosis. FR $\alpha$ is localised on the basolateral and apical brush border surfaces of the choroid plexus epithelium. 5-MTHF transport into the brain parenchyma is mediated by exosomes that transport 5-MTHFladen FR $\alpha$ molecules, traversing the cytosol of choroid plexus epithelial cells from the basolateral to the apical membranes, before being secreted into the CSF at the apical brush border. This suggests that the secretion of $\mathrm{FR} \alpha$ is the major transport pathway for cerebral folate [5, 21, 22].

In both 'auto-immune' and genetic cases, improvement in neurological function and development was achieved by high dose oral and intravenous treatment with folinic acid, an activated folate that is not transporter-dependent. In genetic FOLR1 (folate receptor alpha) deficiency, treatment with oral folinic acid supplementation at doses ranging from $0.5-7 \mathrm{mg} / \mathrm{kg} /$ day has been reported to result in improvement in seizures, neuro-imaging changes and CSF 5 -MTHF levels, although the response may vary depending on the duration of symptoms and timing of treatment initiation [5]. 
Secondary CSF-5-MTHF deficiency in patients with mitochondrial diseases results from deficient activity of FR $\alpha$ due to mitochondrial dysfunction of the choroid plexus epithelium, where vesicle-mediated FR $\alpha$ is located (see above). Low CSF levels of folate in Kearns-Sayre syndrome (KSS) have been known since the 1980s and before the discovery of the causal mtDNA deletions [23]. Hasselmann et al. reported on a girl of three and a half with Alpers disease, a distinct CSF 5-MTHF deficiency, and raised inflammatory markers in CSF along with elevated antibodies against folate receptor [24]. The child died two years later due to disease progression, but treatment with high-dose folinic acid had by then resulted in cessation of status epilepticus and improved neurological function. Grapp et al. [22] not only showed an absence of FR $\alpha$ in the CSF of patients with biallelic FOLR1 variants; they also demonstrated a correlation between the reduction of FR $\alpha$ and a low 5-MTHF concentration in CSF in patients suffering from KSS (Figure 2). Tanji et al. studied the brains in two deceased patients with KSS and described a unique oncocytic transformation of the choroid plexus epithelium. They postulated that these abnormalities were due to the accumulation of abnormal deleted mtDNA which may affect the integrity of the choroid plexus, thereby affecting the blood-brain barrier with loss of FR $\alpha$, presumably via decreased exosome-shuttling, leading to low 5-MTFH levels and raised CSF protein [23]. Elevated CSF protein has been demonstrated in $70 \%$ of patients with POLG-related epilepsies and the level of CSF protein correlates with the severity of the clinical course [25].

Our patient with POLG-related disease appears to be a further case with loss of CSF FR $\alpha$, secondary to mitochondrial dysfunction. It is not possible to determine which part of his symptoms was due to the mitochondriopathy and which to the secondary folate deficiency. However, there is an overlap of symptoms between both conditions. Therefore, his neurological disability may have been aggravated by the impaired folate transport. He was treated with high dose oral and intravenous folinic acid for more than 18 months. A partial recovery of neurological symptoms under treatment with folinic acid supports the notion that cerebral folate deficiency may have contributed to his neurological symptoms. However, despite an objective improvement in neurological function, the boy eventually died because of the severe gastrointestinal manifestations and its complications.

\section{Conclusion}

Pathogenic variants in the $P O L G$ gene can lead to an extremely wide spectrum of neurological and other symptoms, not infrequently missing hepatopathy and lactic acidosis. We therefore recommend early analysis of the POLG gene in such cases. We suspect a paternal germline variant in this family, which is very rare in autosomal-recessive diseases. Significant deficiency of CSF 5-MTHF may contribute to the neurological manifestations, which can be partially rescued by high dose treatment with folinic acid.

\section{Acknowledgements}

The parents have given written consent to publication of the clinical and genetic data, so no additional approval of an ethicscommittee was necessary.

\section{Competing interests}

The author(s) declare that they have no competing interests.

\section{Author contributions}

All authors have made substantial contributions to conception and design, or acquisition of data, or analysis and interpretation of data; have been involved in drafting the manuscript or revising it critically for important intellectual content; and have given their final approval of the version to be published.

This is an Open Access article distributed under the terms of the Creative Commons Attribution License (http://creativecommons.org/licenses/by/4.0), which permits unrestricted use, distribution, and reproduction in any medium, provided the original work is properly credited. The Creative Commons Public Domain Dedication waiver (http://creativecommons.org/publicdomain/zero/l.0/) applies to the data made available in this article, unless otherwise stated.

\section{Cite this article as:}

Korinthenberg R. et al. (2020). Alpers- and MNGIE-like disease with disturbed CSF folate transport and an unusual mode of genetic transmission of POLG mutations: a case report. Journal of the International Child Neurology Association, 20(216). https://doi.org/10.17724/jicna.2020.216

\section{References}

[1] Hikmat O, Tzoulis C, Chong WK, Chentouf L, Klingenberg $\mathrm{C}$, Fratter $\mathrm{C}$, et al. The clinical spectrum and natural history of early-onset diseases due to DNA polymerase gamma mutations. Genetics in Medicine. 2017;19(11):1217-1225. PubMed.

[2] Rahman S, Copeland WC. POLG-related disorders and their neurological manifestations. Nature Reviews Neurology. 2018;15(1):40-52. PubMed.

[3] Chinnery PF, Zeviani M. $155^{\text {th }}$ ENMC workshop: Polymerase gamma and disorders of mitochondrial DNA synthesis, 21-23 September 2007, Naarden, The Netherlands. Neuromuscular Disorders. 2008;18(3):259-267. PubMed.

[4] Hikmat O, Naess K, Engvall M, Klingenberg C, Rasmussen M, Tallaksen CM, et al. Simplifying the clinical classification of polymerase gamma (POLG) disease based on age of onset; studies using a cohort of 155 cases. Journal of Inherited Metabolic Disease. 2020;43(4):726-736. PubMed. 
[5] Pope S, Artuch R, Heales S, Rahman S. Cerebral folate deficiency: Analytical tests and differential diagnosis. Journal of Inherited Metabolic Disease. 2019;42(4):655-672. PubMed.

[6] Steinfeld R, Grapp M, Kraetzner R, Dreha-Kulaczewski S, Helms G, Dechent P, et al. Folate Receptor Alpha Defect Causes Cerebral Folate Transport Deficiency: A Treatable Neurodegenerative Disorder Associated with Disturbed Myelin Metabolism. The American Journal of Human Genetics. 2009;85(3):354-363. PubMed.

[7] Gempel K, Topaloglu H, Talim B, Schneiderat P, Schoser $\mathrm{BGH}$, Hans VH, et al. The myopathic form of coenzyme Q10 deficiency is caused by mutations in the electrontransferring-flavoprotein dehydrogenase (ETFDH) gene. Brain. 2007;130(8):2037-2044. PubMed.

[8] Bulst S, Abicht A, Holinski-Feder E, Muller-Ziermann S, Koehler U, Thirion $C$, et al. In vitro supplementation with dAMP/dGMP leads to partial restoration of mtDNA levels in mitochondrial depletion syndromes. Human Molecular Genetics. 2009;18(9):1590-1599. PubMed.

[9] AlJabri MF, Kamal NM, Halabi A, Korbi H, Alsayyali MMA, Alzahrani YA. Lethal neonatal mitochondrial phenotype caused by a novel polymerase subunit gamma mutation. Medicine. 2018;97(40):e12591. PubMed.

[10] Dai L FF, Liu Z SD, C D, J L, X R, H W. Phenotype and genotype of twelve Chinese children with mitochondrial DNA depletion syndromes. Chin J Pediatr. 2019;57(3):211-216. PubMed.

[11] Han XD, Fang F, Li H, Liu ZM, Shi YQ, Wang JL, et al. Clinical and genetic characteristics of 62 children with mitochondrial epilepsy. Chin J Pediatr. 2019;57(11):844-85. PubMed.

[12] Richards S, , Aziz N, Bale S, Bick D, Das S, et al. Standards and guidelines for the interpretation of sequence variants: a joint consensus recommendation of the American College of Medical Genetics and Genomics and the Association for Molecular Pathology. Genetics in Medicine. 2015;17(5):405-423. PubMed.

[13] Tsiatis AC, Norris-Kirby A, Rich RG, Hafez MJ, Gocke CD, Eshleman JR, et al. Comparison of Sanger Sequencing, Pyrosequencing, and Melting Curve Analysis for the Detection of KRAS Mutations. The Journal of Molecular Diagnostics. 2010;12(4):425-432. PubMed.

[14] de Lange IM, Koudijs MJ, van 't Slot R, Sonsma ACM, Mulder F, Carbo EC, et al. Assessment of parental mosaicism in SCN1A-related epilepsy by single-molecule molecular inversion probes and next-generation sequencing. Journal of Medical Genetics. 2018;56(2):75-80. PubMed.
[15] Møller RS, Liebmann N, Larsen LHG, Stiller M, Hentschel J, Kako N, et al. Parental mosaicism in epilepsies due to alleged de novo variants. Epilepsia. 2019. PubMed.

[16] Anazi S, Al-Sabban E, Alkuraya FS. Gonadal mosaicism as a rare cause of autosomal recessive inheritance. Clinical Genetics. 2013;85(3):278-281. PubMed.

[17] Velho RV, Alegra T, Sperb F, Ludwig NF, Saraiva-Pereira ML, Matte U, et al. A de novo or germline mutation in a family with Mucolipidosis III gamma: Implications for molecular diagnosis and genetic counseling. Molecular Genetics and Metabolism Reports. 2014;1:98-102. PubMed.

[18] Wolf NI, Rahman S, Schmitt B, Taanman JW, Duncan AJ, Harting I, et al. Status epilepticus in children with Alpers' disease caused byPOLG1mutations: EEG and MRI features. Epilepsia. 2009;50(6):1596-1607. PubMed.

[19] Tang S, Dimberg EL, Milone M, Wong LJC. Mitochondrial neurogastrointestinal encephalomyopathy (MNGIE)like phenotype: an expanded clinical spectrum of POLG1 mutations. Journal of Neurology. 2011;259(5):862-868. PubMed.

[20] Ramaekers VT, Rothenberg SP, Sequeira JM, Opladen T, Blau N, Quadros EV, et al. Autoantibodies to Folate Receptors in the Cerebral Folate Deficiency Syndrome. New England Journal of Medicine. 2005;352(19):1985-1991. PubMed.

[21] Grapp M, Just IA, Linnankivi T, Wolf P, Lücke T, Häusler $\mathrm{M}$, et al. Molecular characterization of folate receptor 1 mutations delineates cerebral folate transport deficiency. Brain. 2012;135(7):2022-2031. PubMed.

[22] Grapp M, Wrede A, Schweizer M, Hüwel S, Galla HJ, Snaidero N, et al. Choroid plexus transcytosis and exosome shuttling deliver folate into brain parenchyma. Nature Communications. 2013;4(1). PubMed.

[23] Tanji K, Schon EA, DiMauro S, Bonilla E. Kearns-Sayre syndrome: oncocytic transformation of choroid plexus epithelium. Journal of the Neurological Sciences. 2000;178(1):29-36. PubMed.

[24] Hasselmann O, Blau N, Ramaekers VT, Quadros EV, Sequeira JM, Weissert M. Cerebral folate deficiency and CNS inflammatory markers in Alpers disease. Molecular Genetics and Metabolism. 2010;99(1):58-61. PubMed.

[25] Hikmat O, Naess K, Engvall M, Klingenberg C, Rasmussen M, Tallaksen CME, et al. Elevated cerebrospinal fluid protein inPOLG-related epilepsy: Diagnostic and prognostic implications. Epilepsia. 2018;59(8):1595-1602. PubMed. 